Jacob, K. S. (1999) Mental disorders across cultures: the common issues. International Review of Psychiatry, II, III-II5.

Joel, D., Sathyaseelan, M., Jayakaran, R., et al (2003) Explanatory models of psychosis among community health workers in South India. Acta Psychiatrica Scandinavica, 108, 66-69.

Saravanan, B., Jacob, K. S., Prince, M., et al (2004) Culture and insight revisited. British Journal of Psychiatry, 184, 107-109.

B. Saravanan Institute of Psychiatry, London SE5 8AF, UK

K. S. Jacob Department of Psychiatry, Christian Medical College, Vellore 632002, India.

Email: ksjacob@cmcvellore.ac.in

doi: 10.1192/bjp.189.3.284a

\section{Restarting clozapine following leucopenia or neutropenia}

Dunk et al (2006) report rechallenge with clozapine of people with either treatmentresistant or treatment-intolerant schizophrenia. A proportion of these may lack insight and capacity and may therefore be detained under the Mental Health Act 1983 but the authors do not indicate the proportion of patients in this group. If a person has been compulsorily detained, the treating clinician may require a second opinion from the Mental Health Act Commission. We are interested in whether Dunk et al have any data on this, as in the British National Formulary clozapine is contraindicated in those who have previously developed dyscrasia. The Mental Health Act Commission may not provide a second opinion for drugs that are contraindicated.

Dunk et al report a possible alternative explanation for dyscrasia during first exposure to clozapine in 25 patients. There was no alternative explanation in the remaining 28 patients. An obvious question that arises is whether a patient is more or less likely to develop dyscrasia on rechallenge if they have a history of an alternative explanation. This would be a very useful predictor and would be helpful when discussing the options with the patient prior to rechallenge.

\section{British Medical Association \& Royal}

Pharmaceutical Society (2005) British National Formulary. BMJ Publishing Group \& Pharmaceutical Press.

Dunk, L. R., Annan, L. J. \& Andrews, C. D. (2006) Rechallenge with clozapine following leucopenia or neutropenia during previous therapy. British Journal of Psychiatry, 188, 255-263.

P. Soma, P. C. Naik Lyndon Clinic, Birmingham and Solihull Mental Health NHS Trust, Hobs Meadow, Solihull, West Midlands B92 8PW, UK. Email: prakash.naik@bsmht.nhs.uk

doi: 10.1192/bjp.189.3.285

Authors' reply: The Clozaril Patient Monitoring Service (CPMS) does not record which patients are being treated under the Mental Health Act 1983 and we are therefore unable to say what proportion of patients in our cohort were compulsorily detained. We are not aware of any studies regarding second opinions from the Mental Health Act Commission in patients undergoing rechallenge with clozapine but would be interested to hear of any.

We have re-examined our data to determine whether patients were more or less likely to develop dyscrasia on rechallenge if they had a history of an alternative explanation for the first episode of dyscrasia. Out of 53 patients in the cohort, 25 had an alternative explanation for the first episode and 6 of these $(24 \%)$ developed a second episode on rechallenge. Out of the 28 patients with no alternative explanation for the first episode of dyscrasia, 14 (50\%) experienced dyscrasia on rechallenge. The difference was not significant $(P=0.05914)$. The relative risk of 2.08 indicated that patients with no alternative explanation may be twice as likely to have a second episode of dyscrasia on rechallenge as those with an alternative explanation, but the $95 \%$ confidence interval was 0.98-6.2. We must stress that alternative explanations for dyscrasia may not always be reported to the CPMS, therefore these figures may not represent the true picture and this aspect of our work should be interpreted with caution.

\section{Declaration of interest}

L.D. has undertaken consultancy for Novartis UK and Novartis Australia and received a fee from Novartis Australia for the preparation of this paper; she was formerly employed by Novartis UK. L.A. and C.A. are employed by Novartis UK.

L. R. Dunk Department of Histopathology, Leicester Royal Infirmary, Leicester LEI 5WW, UK. Email: louisa.dunk@btinternet.com
L. Annan, C. Andrews Novartis

Pharmaceuticals UK Ltd, Camberley, UK

doi: 10.II92/bjp.I89.3.285a

\section{Risk factors for coronary heart disease in people with severe mental illness}

Osborn et al (2006) compared risk factors for coronary heart disease (CHD) in people with and without severe mental illness (SMI) in primary care.

A number of points in the results, discussion and conclusions seem unjustified and are potentially misleading. For example, the statement that patients with SMI had a significantly raised CHD risk score is based upon the unadjusted risk. After adjustment for age and gender the odds ratio dropped below the level of statistical significance and fell further to a nonsignificant value of 1.3 (95\% CI $0.7-2.7)$ after considering employment status. The authors' claim that 'we have demonstrated that SMI itself can incur CHD risk, over and above that associated with the socioeconomic deprivation experienced by these patients' is not justified.

This claim is repeated in the abstract: 'excess risk factors for CHD are not wholly accounted for by medication or socioeconomic deprivation'. This statement seems either unproven or reducible to the fact that smoking is more common among people with SMI. Such a conclusion is scarcely novel and clearly does not explain the excess mortality observed in patients with SMI (Joukamaa et al, 2006). The fact that diabetes is both more common among people with SMI and much less explicable in terms of their deprivation or demographics receives relatively little comment, despite having particular relevance for their healthcare needs.

Joukamaa, M., Heliövaara, M., Knekt, P., et al (2006) Schizophrenia, neuroleptic medication and mortality. British Journal of Psychiatry, 188, 122-127.

Osborn, D. P. J., Nazareth, I. \& King, M. B. (2006) Risk for coronary heart disease in people with severe mental illness: cross-sectional comparative study in primary care. British journal of Psychiatry, 188, 27I-277.

C. Gilleard Department of Psychology and Psychotherapies, Springfield University Hospital, Tooting, London SWI7 7DJ, UK. Email: Chris.Gilleard@swlstg-tr.nhs.uk doi: 10.I192/bjp.189.3.285b 